\title{
CAPACITARY TYPE ESTIMATES IN STRONGLY NONLINEAR POTENTIAL THEORY AND APPLICATIONS
}

\author{
Noureddine AISSAOUI
}

\begin{abstract}
In this article a general result on smooth truncation of Riesz and Bessel potentials in Orlicz-Sobolev spaces is given and a capacitary type estimate is presented. We construct also a space of quasicontinuous functions and an alternative characterization of this space and a description of its dual are established. For the Riesz kernel $\mathcal{R}_{m}$, we prove that operators of strong type $(A, A)$, are also of capacitaries strong and weak types $(m, A)$.
\end{abstract}

\section{Introduction}

In this paper, we continue the development of the Potential Theory in Orlicz spaces, called Strongly Nonlinear Potential Theory, studied in [6, $7,8,9,10,11]$.

We begin by establishing, in Theorem 3.5, a general result on smooth truncation of Riesz and Bessel potentials in Orlicz-Sobolev spaces $W^{m} L_{A}\left(R^{N}\right)$ such that $A$ and $A^{*}$ satisfy the $\Delta_{2}$ condition. This result has been proven, in the framework of Lebesgue spaces, first by V. G. Maz'ya for the first order type inequality using truncation in the class $W^{1, p}$. For the Sobolev space $W^{2, p}$, Maz'ya used smooth truncation to get the result. Later, D. R. Adams proved the same result for any integer. After several intermediate stages, the result was proved in the generality by K. Hansson.

As a consequence, by showing the equivalence of some capacities, we obtain, in Theorem 3.7, the capacitary strong type estimates with the aid of the norms defined on Orlicz spaces.

2000 Mathematics Subject Classification: 46E35, 31B15.

Servicio de Publicaciones. Universidad Complutense. Madrid, 2001 
On the other hand, for the Bessel capacity $B_{m, A}^{\prime}$ in the Orlicz space $\mathbf{L}_{A}$, the natural question: "does the quantity $\int_{0}^{\infty} B_{m, A}^{\prime}(\{x:|\psi| \geq t\}) d t$ define a norm on a linear space of functions $\psi$ on $R^{N}$ ?", leads us to construct the space $\mathbf{L}_{A}\left(B_{m, A}^{\prime}\right)$ of quasicontinuous functions "integrable with respect to capacity", with the aid of the Orlicz space $\mathbf{L}_{A}$ and the capacity $B_{m, A}^{\prime}$. We prove, for reflexive Orlicz spaces $\mathbf{L}_{A}$, that $\mathbf{L}_{A}\left(B_{m, A}^{\prime}\right)$ contains the potential space $\mathbf{L}_{m, A}$. We give in Theorem 4.6, for reflexive Orlicz spaces $\mathbf{L}_{A}$, a characterization of $\mathbf{L}_{A}\left(B_{m, A}^{\prime}\right)$ as the space of quasicontinuous functions $\psi$ which satisfy the following boundedness condition: $\int_{0}^{\infty} B_{m, A}^{\prime}(\{x:|\psi(x)| \geq t\}) d t<\infty$. As a consequence, if the potential space $\mathbf{L}_{m, A}$ is imbedded in a Banach space $\mathbf{B}$ which is partially ordered in the sense that $u, v \in \mathbf{B}$ and $|u(x)| \leq|v(x)|$ everywhere implies that $\|u\|_{\mathbf{B}} \leq\|v\|_{\mathbf{B}}$ for a norm of $\mathbf{B}$, then $\mathbf{B}$ contains $\mathbf{L}_{A}\left(B_{m, A}^{\prime}\right)$.

We characterize also, in Theorem 4.8 , the dual $\mathbf{L}_{A}\left(B_{m, A}^{\prime}\right)^{*}$ of $\mathbf{L}_{A}\left(B_{m, A}^{\prime}\right)$, when $A$ verifies the $\Delta_{2}$ condition, as the space of measures whose total variation belongs to the dual of the potential space $\mathbf{L}_{m, A}$.

Finally, in the case of the Riesz kernel $\mathcal{R}_{m}$, we prove in Proposition 5.1 , that operators of strong type $(A, A)$, are also of capacitary strong type $(m, A)$ if $A$ and $A^{*}$ satisfy the $\Delta_{2}$ condition, and of capacitary weak type $(m, A)$ for any $\mathrm{N}$-function $A$.

\section{Preliminaries}

\subsection{Orlicz spaces}

An $N$-function is a continuous convex and even function $A$ defined on $R$, verifying $A(t)>0$ for $t>0, \lim _{t \rightarrow 0} \frac{A(t)}{t}=0$ and $\lim _{t \rightarrow+\infty} \frac{A(t)}{t}=+\infty$.

We have the representation $A(t)=\int_{0}^{|t|} a(x) d x$, where $a: R^{+} \rightarrow R^{+}$ is non-decreasing, right continuous, with $a(0)=0, a(t)>0$ for $t>0$, $\lim _{t \rightarrow 0^{+}} a(t)=0$ and $\lim _{t \rightarrow+\infty} a(t)=+\infty$.

The N-function $A^{*}$ conjugate to $A$ is defined by $A^{*}(t)=\int_{0}^{|t|} a^{*}(x) d x$, where $a^{*}$ is given by $a^{*}(s)=\sup \{t: a(t) \leq s\}$.

Let $A$ be an N-function and $\Omega$ an open set in $R^{N}$. We note $\mathcal{L}_{A}(\Omega)$ the set, called an Orlicz class, of measurable functions $f$, on $\Omega$, such 
that

$$
\rho(f, A, \Omega)=\int_{\Omega} A(f(x)) d x<\infty .
$$

Let $A$ and $A^{*}$ be two conjugate $\mathrm{N}$-functions and let $f$ be a measurable function defined almost everywhere in $\Omega$. The Orlicz norm of $f,\|f\|_{A, \Omega}$ or $\|f\|_{A}$ if there is no confusion, is defined by

$$
\|f\|_{A}=\sup \left\{\int_{\Omega}|f(x) g(x)| d x: g \in \mathcal{L}_{A^{*}}(\Omega) \text { and } \rho\left(g, A^{*}, \Omega\right) \leq 1\right\} .
$$

The set $\mathbf{L}_{A}(\Omega)$ of measurable functions $f$, such that $\|f\|_{A}<\infty$ is called an Orlicz space. When $\Omega=R^{N}$, we set $\mathbf{L}_{A}$ in place of $\mathbf{L}_{A}\left(R^{N}\right)$.

The Luxemburg norm \|\|$f|\||_{A, \Omega}$ or $\||f|\|_{A}$ if there is no confusion, is defined in $\mathbf{L}_{A}(\Omega)$ by

$$
\|\mid f\|_{A}=\inf \left\{r>0: \int_{\Omega} A\left(\frac{f(x)}{r}\right) d x \leq 1\right\} .
$$

Orlicz and Luxemburg norms are equivalent. More precisely, if $f \in$ $\mathbf{L}_{A}(\Omega)$, then

$$
\left\|| | f \left|\| _ { A } \leq \| f \left\|_{A} \leq 2\left|\|f \mid\|_{A} .\right.\right.\right.\right.
$$

Let $A$ be an $\mathrm{N}$-function. We say that $A$ verifies the $\Delta_{2}$ condition if there is a constant $C>0$ such that $A(2 t) \leq C A(t)$ for all $t \geq 0$.

Recall that $A$ verifies the $\Delta_{2}$ condition if and only if $\mathcal{L}_{A}=\mathbf{L}_{A}$. Moreover $\mathbf{L}_{A}$ is reflexive if and only if $A$ and $A^{*}$ verify the $\Delta_{2}$ condition.

Note that if $A$ verifies the $\Delta_{2}$ condition, $\int A\left(f_{i}(x)\right) d x \rightarrow 0$ as $i \rightarrow \infty$ if and only if $\left\|\mid f_{i}\right\| \|_{A} \rightarrow 0$ as $i \rightarrow \infty$.

Let $m \in N$. The Orlicz-Sobolev space $W^{m} L_{A}(\Omega)$ is the space of real functions $f$, such that $f$ and its distributional derivatives up to the order $m$, are in $L_{A}(\Omega)$.

$W^{m} L_{A}(\Omega)$ is a Banach space equipped with the norm

$$
\left\||| f||_{m, A}=\sum_{|i| \leq m}\right\||| D^{i} f \mid \|_{A}, f \in W^{m} L_{A}(\Omega) .
$$

Let $W^{-m} L_{A^{*}}(\Omega)$ denote the space of distributions on $\Omega$, which can be written as sums of derivatives up to the order $m$ of functions in $L_{A^{*}}(\Omega)$. It is a Banach space under the usual quotient norm. 
If $A$ and $A^{*}$ satisfy the $\Delta_{2}$ condition, the dual of $W^{m} L_{A}\left(R^{N}\right)$ coincides with $W^{-m} L_{A^{*}}\left(R^{N}\right)$.

We recall the following results. Let $A$ be an $\mathrm{N}$-function and $a$ its derivative. Then, from [19], the following statements are equivalent

i) $A$ verifies the $\Delta_{2}$ condition.

ii) $\forall r>1, \exists k=k(r):(\forall t \geq 0, A(r t) \leq k A(t))$

iii) $\exists \alpha>1:(\forall t \geq 0, t a(t) \leq \alpha A(t))$

$i v) \exists \beta>1:\left(\forall t \geq 0, t a^{*}(t) \geq \beta A^{*}(t)\right)$

v) $\exists d>0:\left(\forall t \geq 0,\left(\frac{A^{*}(t)}{t}\right)^{\prime} \geq d \frac{a^{*}(t)}{t}\right)$.

Moreover $\alpha$ in iii) and $\beta$ in $i v$ ) can be chosen such that $\alpha^{-1}+\beta^{-1}=1$. We note $\alpha(A)$ the smallest $\alpha$ such that iii) holds.

For more details on the theory of Orlicz spaces, see [5, 19, 20, 21, $23]$.

\subsection{Capacity, Bessel potentials and Quasicontinuity}

We define a capacity as an increasing positive set function $C$ given on a $\sigma$-additive class of sets $\Gamma$, which contains compact sets and such that $C(\emptyset)=0$ and $C\left(\bigcup_{i \geq 1} X_{i}\right) \leq \sum_{i \geq 1} C\left(X_{i}\right)$ for $X_{i} \in \Gamma, i=1,2, \ldots$

Let $k$ be a positive and measurable function on $R^{N}$ and let $A$ be an $\mathrm{N}$-function. For $X \subset R^{N}$, we define

$$
C_{k, A}^{\prime}(X)=\inf \left\{\|\| f \|_{A}: f \in \mathbf{L}_{A}^{+} \text {and } k * f \geq 1 \text { on } X\right\},
$$

and

$$
C_{k, A}(X)=A\left(C_{k, A}^{\prime}(X)\right),
$$

where $k * f$ is the usual convolution and $\mathbf{L}_{A}^{+}$is the set of positive elements in $\mathbf{L}_{A}$.

From $[10] C_{k, A}^{\prime}$ is a capacity.

If a statement holds except on a set $X$ where $C_{k, A}(X)=0$, then we say that the statement holds $C_{k, A}$-quasieverywhere (abbreviated $C_{k, A}-q . e$. or $(k, A)-q . e$. if there is no confusion).

For $m>0$, the Bessel kernel $\mathcal{G}_{m}$ is defined through its Fourier transform $\mathfrak{F}\left(\mathcal{G}_{m}\right)$ as $\left[\mathfrak{F}\left(\mathcal{G}_{m}\right)\right](x)=(2 \pi)^{-\frac{N}{2}}\left(1+|x|^{2}\right)^{-\frac{m}{2}}$ where $[\mathfrak{F}(f)](x)=$ $(2 \pi)^{-\frac{N}{2}} \int f(y) e^{-i x y} d y$ for $f \in \mathbf{L}^{1}$. 
The Bessel kernel $\mathcal{G}_{m}$ is a positive and Lebesgue integrable function. Moreover, for any $r$ and $s, \mathcal{G}_{r+s}=\mathcal{G}_{r} * \mathcal{G}_{s}$.

For more details on Bessel kernels, see [12, 13, 24].

We note $\mathcal{R}_{m}(x)=|x|^{m-N}$ the Riesz kernel. $C_{\mathcal{R}_{m}, A}^{\prime}$

We put $B_{m, A}=C_{\mathcal{G}_{m}, A}, B_{m, A}^{\prime}=C_{\mathcal{G}_{m}, A}^{\prime}, R_{m, A}=C_{\mathcal{R}_{m}, A}$ and $R_{m, A}^{\prime}=$

Let $A$ be an $\mathrm{N}$-function and $m \in R$. We define the space of Bessel potentials $\mathbf{L}_{m, A}$ by

$$
\mathbf{L}_{m, A}=\left\{\psi=\mathcal{G}_{m} * f: f \in \mathbf{L}_{A}\right\},
$$

and a norm on $\mathbf{L}_{m, A}$ by

$$
\||\psi|\|_{m, A}=\|\| f \|_{A} \text { if } \psi=\mathcal{G}_{m} * f .
$$

From [14] we know that if $m \in N$, then $\mathbf{L}_{m, A}=W^{m} L_{A}\left(R^{N}\right)$ with equivalent norms.

Recall the following fact (see [19]) : If $A$ verifies the $\Delta_{2}$ condition and if $F$ is a continuous linear functional over $\mathbf{L}_{A}$, then there is a unique $v \in \mathbf{L}_{A^{*}}$ such that

$$
F(u)=\langle u, v\rangle=\int u(x) v(x) d x, \forall u \in \mathbf{L}_{A} .
$$

This implies, for $A$ verifying the $\Delta_{2}$ condition, that $\left(\mathbf{L}_{m, A}\right)^{*}=$ $\mathbf{L}_{-m, A^{*}}$. The proof is exactly the same as the one in [15] relative to Lebesgue classes.

We recall the general definition of quasicontinuity.

Definition 2.1. Let $\mathcal{C}$ be a capacity on $R^{N}$ and let $f$ be a function defined $\mathcal{C}-$ q.e. on $R^{N}$ or on some open subset of $R^{N}$. Then $f$ is said to be $\mathcal{C}$-quasicontinuous if for every $\varepsilon>0$, there is an open set $O$ such that $\mathcal{C}(O)<\varepsilon$ and $\left.f\right|_{O^{c}} \in C\left(O^{c}\right)$.

In other words, the restriction of $f$ to the complement of $O$ is continuous in the induced topology.

For Bessel capacity $B_{m, A}^{\prime}$, we write $(m, A)$-quasicontinuous in place of $B_{m, A^{\prime}}^{\prime}$-quasicontinuous.

Recall the following result. See $[9]$ 
Theorem 2.1. Let $A$ be any $N$-function. Let $f$ and $g$ be two $(m, A)$ quasicontinuous functions, $m>0$. Suppose that $f(x)=g(x)$ almost everywhere. Then $f(x)=g(x)(m, A)$-quasieverywhere.

Note $\mathfrak{M}=\mathfrak{M}\left(R^{N}\right)$ the vector space of Radon measures on $R^{N}$. Then any $(m, A)$-quasicontinuous function $f$ is measurable with respect to any $\mu \in \mathfrak{M}$ that is absolutely continuous with respect to $(m, A)$-capacity in the sense that $|\mu|(E)=0$ for every Borel set $E$ with $B_{m, A}(E)=0$. In fact, from Definition 2.1 and the Tietze extension theorem, it follows that a quasicontinuous function is the pointwise limit of continuous functions outside some $G_{\delta}$ set of $(m, A)$-capacity zero.

Thus $\int_{R^{N}} f d \mu$ is well defined as soon as $\int_{R^{N}}|f| d|\mu|<\infty$. It follows from Theorem 2.1 that $\int_{R^{N}} f d \mu=\int_{R^{N}} g d \mu$ even if we only know that $f=g$ a.e., in addition to being quasicontinuous.

On the other hand, for any positive measure $\mu$ and any Borel set $E$, we have for all $f \in \mathbf{L}_{A}^{+}$such that $k * f \geq 1$ on $E$,

$$
\mu(E) \leq \int\left(\mathcal{G}_{m} * f\right) d \mu \leq \int\left(\mathcal{G}_{m} * \mu\right) . f d x \leq\|\| f \mid\|\|_{A} \cdot\left\|\mathcal{G}_{m} * \mu\right\|_{A^{*}}
$$

Thus

$$
\mu(E) \leq B_{m, A}(E) \cdot\left\|\mathcal{G}_{m} * \mu\right\|_{A^{*}}
$$

Hence all $(m, A)$-quasicontinuous functions $f$ are measurable with respect to all $\mu$ such that $|\mu| \in\left(\mathbf{L}_{m, A}\right)^{*}$, (respectively $|\mu| \in \mathbf{L}_{-m, A^{*}}$ if $A$ verifies the $\Delta_{2}$ condition) and equality $(m, A)$-quasieverywhere implies equality $\mu$-a.e.

We know also from [9] that if $A$ verifies the $\Delta_{2}$ condition, then every element in $\mathbf{L}_{m, A}$ has an $(m, A)$-quasicontinuous representative. Thus if $\psi \in \mathbf{L}_{m, A}$, there is $f \in \mathbf{L}_{A}$ such that $\mathcal{G}_{m} * f$ is $(m, A)$-quasicontinuous and $\psi=\mathcal{G}_{m} * f$.

Hence, for any $\mu \in \mathfrak{M}\left(R^{N}\right)$ such that $|\mu| \in \mathbf{L}_{-m, A^{*}}$, we get

$$
\int_{R^{N}}|\psi| d|\mu| \leq \int_{R^{N}} \mathcal{G}_{m} *|f| d|\mu|
$$

By Fubini's Theorem

$$
\int_{R^{N}} \mathcal{G}_{m} *|f| d|\mu|=\int_{R^{N}}|f| \cdot\left(\mathcal{G}_{m} *|\mu|\right) d x .
$$

Hölder inequality in Orlicz spaces gives 


$$
\int_{R^{N}}|f| \cdot\left(\mathcal{G}_{m} *|\mu|\right) d x \leq|||f|||_{A} \cdot|| \mathcal{G}_{m} *|\mu|\|\|_{A^{*}}<\infty .
$$

Hence $\int_{R^{N}}|\psi| d|\mu|<\infty$.

We deduce for such measure, that $\psi$ belongs to $\mathbf{L}^{1}(|\mu|)$, and that $\mathbf{L}_{m, A}$ is continuously imbedded in $\mathbf{L}^{1}(|\mu|)$. Moreover, in this case the duality between $\mathbf{L}_{-m, A^{*}}$ and $\mathbf{L}_{m, A}$ is given by

$$
\langle\mu, \psi\rangle=\int_{R^{N}} \psi d \mu=\int_{R^{N}}\left(\mathcal{G}_{m} * \mu\right) \cdot f d x .
$$

This result is extended in Theorem 4.8 below.

Notice also that if $\mu \in \mathfrak{M}^{+}\left(R^{N}\right) \cap \mathbf{L}_{-m, A^{*}}$, then $\mu(E)=0$ for every $\mu$-measurable set $E$ such that $B_{m, A}(E)=0$.

In [7] we have extended this result to signed measures.

In this paper, the letter $C$ will denote various constants which may differ from one formula to the next one even within a single string of estimates. We make no attempt to obtain the best values for these constants.

\section{A capacitary type estimate}

In this section we begin with some lemmas

Lemma 3.1. Let $A$ be an $N$-function, $f \in \mathbf{L}_{A}$ and $\Omega$ a measurable set in $R^{N}$. Then

$$
\|f\|_{A, \Omega}=\left\|f .1_{\Omega}\right\|_{A} \text { and }\|\| f\left|\left\|_{A, \Omega}=\right\|\right|\left|f . \mathbf{1}_{\Omega}\right| \|_{A} .
$$

Proof. We omit the proof.

Lemma 3.2 Let $A$ be an $N$-function, $f \in \mathbf{L}_{A}$ and $\left(\Omega_{j}\right)_{j}$ a sequence of measurable sets such that $\Omega_{j} \cap \Omega_{l}=\emptyset$ if $j \neq l$. Then

$$
\sum_{j}\|f\|_{A, \Omega_{j}} \leq\|f\|_{A} \text {, and } \sum_{j}\|\| f\|\|_{A, \Omega_{j}} \leq 4\left|\|f \mid\|_{A} .\right.
$$

Proof. For $\varepsilon>0$ and $j \in N$, there is $\varphi_{j}$ such that

$$
\int_{\Omega_{j}} A^{*}\left(\varphi_{j}(x)\right) d x \leq 1 \text { and }\|f\|_{A, \Omega_{j}} \leq \int_{\Omega_{j}} f \cdot \varphi_{j}(x) d x+\frac{\varepsilon}{2^{j}} .
$$


Define $\psi_{j}=\varphi_{j} . \mathbf{1}_{j}$. Then $\int A^{*}\left(\psi_{j}(x)\right) d x \leq 1$ and $\|f\|_{A, \Omega_{j}} \leq$ $\int f \cdot \psi_{j}(x) d x+\frac{\varepsilon}{2^{j}}$.

Let $\psi=\sup \psi_{j}$. Since $A^{*}$ is non decreasing, the Beppo-Levi Theorem gives

$$
\int\left(A^{*} \circ \psi\right)(x) d x=\varlimsup \lim \int\left(A^{*} \circ \psi_{j}\right)(x) d x \leq 1 .
$$

Moreover $\sum_{j}\|f\|_{A, \Omega_{j}} \leq \sum_{j} \int_{\Omega_{j}} f \cdot \varphi_{j}(x) d x+\varepsilon \leq \int f . \psi(x) d x+\varepsilon$.

Hence $\sum_{j}\|f\|_{A, \Omega_{j}} \leq\|f\|_{A}$.

The second inequality is a consequence of the equivalence between Orlicz and Luxemburg norms.

Let $\mathcal{M}$ be the Hardy-Littlewood maximal operator. Recall the following Lemma..

Lemma 3.3. [9] Let $A$ be any $N$-function. For all multiindices $\xi$ such that $|\xi|<m<N$, there is a constant $C$ such that for all $f \in \mathbf{L}_{A}$ and for almost every $x$,

$$
\left|D^{\xi}\left(\mathcal{G}_{m} * f\right)(x)\right| \leq C \mathcal{M} f(x)^{\frac{|\xi|}{m}}\left(\mathcal{G}_{m} *|f|(x)\right)^{1-\frac{|\xi|}{m}} .
$$

For Riesz kernel, we have the correspondent result which is very simple to verify.

Lemma 3.4. Let $A$ be any $N$-function. For all multi-indices $\xi$ such that $|\xi|<m<N$, there is a constant $C$ such that for all $f \in \mathbf{L}_{A}$ and for almost every $x$,

$$
\left|D^{\xi}\left(\mathcal{R}_{m} * f\right)(x)\right| \leq C \mathcal{M} f(x)^{\frac{|\xi|}{m}}\left(\mathcal{R}_{m} *|f|(x)\right)^{1-\frac{|\xi|}{m}} .
$$

Remark 3.1. From [8] we know that if $A$ verifies the $\Delta_{2}$ condition and if $\alpha=\alpha(A)$ is such that $m<N / \alpha$, then all non empty balls are of strictly positive $R_{m, A}$ capacity. Hence, the restriction $m<N / \alpha$ is essential when working with Riesz capacity $R_{m, A}^{\prime}$. Note that this is the same restriction as in the case of $\mathbf{L}^{p}$.

Theorem 3.5. Let $A$ be an $N$-function such that $A$ and $A^{*}$ verify the $\Delta_{2}$ condition, $\alpha=\alpha(A)$ and $m$ is a positive integer. Let $T_{j \in \mathbf{Z}}$ be a 
doubly infinite sequence of $C^{m}(R)$ functions identically zero for $t<0$ with $T_{j}^{\prime}$ having disjoints supports in $(0, \infty)$ and such that

$$
\sup _{t>0}\left|t^{k-1} T_{j}^{(k)}(t)\right| \leq L<\infty, k=0,1, \ldots, m
$$

Then for all $f \in \mathbf{L}_{A}^{+}$, there is a constant $C$ depending only on $N, m, L$ and $A$ such that

$$
\sum_{j}\left\|| | D ^ { \beta } T _ { j } ( \mathcal { S } _ { m } * f ) \left|\left\|_{A} \leq C\left|\|f \mid\|_{A},\right.\right.\right.\right.
$$

where $\beta$ is a multi-index such that $|\beta|=m$, and $\mathcal{S}_{m}$ is either $\mathcal{G}_{m}$ if $m$ is a positive integer, or $\mathcal{R}_{m}$ if $m$ is a positive integer such that $m<N / \alpha$.

Proof. Let $g=\mathcal{S}_{m} * f$, and $|\beta|=m$. Then

$$
D^{\beta}\left(T_{j} \circ g\right)=\sum_{k=1}^{m} T_{j}^{(k)} \circ g \sum c_{\beta} D^{\beta_{1}} g \ldots D^{\beta_{k}} g,
$$

where the last sum is for the multi-indices $\left\{\beta_{1}, \ldots, \beta_{k}\right\}$ such that $\beta_{1}+$ $\ldots+\beta_{k}=\beta$. The value of the coefficients $c_{\beta}$ is of no importance.

Let $\Omega_{j}$ be the support of $T_{j}^{\prime} \circ g$. Then $\Omega_{j} \cap \Omega_{l}=\emptyset$ if $j \neq l$.

By hypothesis $\left|D^{\beta}\left(T_{j} \circ g\right)\right| \leq C L \sum_{k=1}^{m} g^{1-k} \sum\left|D^{\beta_{1}} g \ldots D^{\beta_{k}} g\right| . \mathbf{1}_{\Omega_{j}}$

For $k>1$, we estimate these derivatives with the aid of the two previous Lemmas. We get

$$
\begin{gathered}
\left|D^{\beta_{k}} g\right| \mathbf{1}_{\Omega_{j}} \leq C \mathcal{M} f(x)^{\frac{\left|\beta_{k}\right|}{m}}\left(\mathcal{S}_{m} *|f|(x)\right)^{1-\frac{\left|\beta_{k}\right|}{m}} \cdot \mathbf{1}_{\Omega_{j}} \\
\text { Since } \sum_{l=1}^{k}\left(1-\frac{\beta_{l}}{m}\right)=k-\frac{|\beta|}{m}=k-1 \text {, we obtain } \\
\sum_{k=2}^{m} g^{1-k} \sum\left|D^{\beta_{1}} g \ldots D^{\beta_{k}} g\right| \mathbf{1}_{\Omega_{j}} \leq C \sum_{k=2}^{m} g^{1-k}(\mathcal{M} f) \cdot g^{k-1}=C(\mathcal{M} f) \cdot \mathbf{1}_{\Omega_{j}}
\end{gathered}
$$

By adding the term for $k=1$, we get

$$
\left|D^{\beta}\left(T_{j} \circ g\right)\right| \leq C L(\mathcal{M} f) \mathbf{1}_{\Omega_{j}}+\left|D^{\beta} g\right| . \mathbf{1}_{\Omega_{j}} .
$$

By Lemma 3.1 
$\|\left|(\mathcal{M} f) \mathbf{1}_{\Omega_{j}}\right|||_{A}=|||\mathcal{M} f|||_{A, \Omega_{j}}$ and $\left\|\left|\left(D^{\beta} g\right) . \mathbf{1}_{\Omega_{j}}\right|||_{A}=\left|\| D^{\beta} g\right|||_{A, \Omega_{j}}\right.$.

And by Lemma 3.2

$$
\sum_{j}\left|\left\|\mathcal { M } f \left|\left\|_{A, \Omega_{j}} \leq 4\left|\|\mathcal{M} f \mid\|_{A} \text { and } \sum_{j}\left\|\left|D^{\beta} g\|\|_{A, \Omega_{j}} \leq 4\right|\right\| D^{\beta} g\|\|_{A} .\right.\right.\right.\right.\right.
$$

By [16], $\left.\||\mathcal{M} f|\|\right|_{A} \leq C\left|\|f \mid\| \|_{A}\right.$, and from [14] we have $\left\|\mid D^{\beta} g\right\| \|_{A} \leq$ $C\left|\left\|f|\||_{A}\right.\right.$.

This completes the proof.

Let $m$ be a positive integer. We define for a compact set $K$ in $R^{N}$,

$$
\begin{gathered}
\Gamma_{m, A}(K)= \\
\inf \left\{\sum_{|\beta|=m}\left|\left\|D^{\beta} g \mid\right\| \|_{A}: g \in D\left(R^{N}\right), g \equiv 1 \text { in a neighborhood of } K\right\} .\right.
\end{gathered}
$$

We extend the definition of $\Gamma_{m, A}$ to all the class of all subsets of $R^{N}$ as an inner capacity. Hence for any $X \subset R^{N}, \Gamma_{m, A}(X)$ is defined as

$$
\Gamma_{m, A}(X)=\sup \left\{\Gamma_{m, A}(K): K \text { compact and } K \subset X\right\} .
$$

Since $\Gamma_{m, A}$ is monotone, this definition agrees with our first one when $X$ is compact. [4].

The $\mathbf{L}^{p}$ version of the following Lemma can be found in [1] and in

Lemma 3.6. Let $A$ be an $N$-function such that $A$ and $A^{*}$ verify the $\Delta_{2}$ condition, $m$ a positive integer such that $m<N / \alpha$. Then there is a positive constant $C$ such that

$$
C^{-1} R_{m, A}^{\prime}(K) \leq \Gamma_{m, A}(K) \leq C R_{m, A}^{\prime}(K),
$$

for all compact $K, C$ independent of $K$.

Proof. We adapt Adams proof in [1] relative to $\mathbf{L}^{p}$ Lebesgue spaces to our case.

We begin by proving the first inequality. Let $g \in D\left(R^{N}\right), g \equiv 1$ in a neighborhood of $K$. From [24] we have the representation for $g \in D\left(R^{N}\right)$

$$
g(x)=\sum_{|\beta|=m} c_{\beta} K_{\beta} * D^{\beta} g(x),
$$


where $K_{\beta}$ is the convolution operator $K_{\beta^{1}} * \ldots * K_{\beta^{N}}, \beta=\left(\beta^{1}, \ldots, \beta^{N}\right)$, $K_{\beta^{j}}$ is the convolution operator whose kernel is $\omega_{N-1}^{-1} \cdot x_{j}|x|^{-N}$ convoled $\beta^{j}$-times.

We get $|g(x)| \leq C \mathcal{R}_{m} *\left(\sum_{|\beta|=m}\left|D^{\beta} g\right|\right)(x)$.

Set $f=C\left(\sum_{|\beta|=m}\left|D^{\beta} g\right|\right)$. We have $\mathcal{R}_{m} * f \geq 1$ on $K$ and

$$
R_{m, A}^{\prime}(K) \leq\||| f \mid\|\left\|_{A} \leq C \sum_{|\beta|=m}\right\|\left\|D^{\beta} g\right\| \|_{A} .
$$

This implies the first inequality.

For the second inequality, we apply Theorem 3.5 for one $J$-function defined by $J(t) \equiv 0$, for $t \leq 1 / 2$, and $J(t) \equiv 1$, for $t \geq 1$, and $J \in C^{\infty}(R)$ otherwise.

Let $\varphi \in D\left(R^{N}\right)^{+}$be such that $\operatorname{supp} \varphi \subset B(0,1)$ and $\int \varphi(x) d x=1$.

Set $\varphi_{h}(x)=h^{-N} \varphi(x / h), h>0$. Choose an open bounded set $G \supset$ $K$ and any $f \in \mathbf{L}_{A}^{+}$such that $\mathcal{R}_{m} * f \geq 1$ on $G$.

Then for sufficiently small $h, \varphi_{h} *\left(\mathcal{R}_{m} * f\right) \geq 1$ on $G_{1}$, where $K \subset$ $G_{1} \subset G, G_{1}$ an open with $\overline{G_{1}} \subset G$. $G_{1}$.

Set $g(x)=J \circ\left(\mathcal{R}_{m} *\left(\varphi_{h} * f\right)\right)(x)$. Clearly $g \in D\left(R^{N}\right)$ and $g \equiv 1$ on

By Theorem 3.5

$$
\Gamma_{m, A}(K) \leq \sum_{|\beta|=m}\left|\| D ^ { \beta } g \| \left\|_{A} \leq C\left|\left\|\varphi_{h} * f \mid\right\| \|_{A}\right.\right.\right.
$$

By [14 or 22], we know that $\left|\left\|\varphi_{h} * f\left|\left\|\left.\right|_{A} \leq\right\| \varphi_{h}\left\|_{1} \cdot\left|\left\|f\left|\left\|\left.\right|_{A}=\right\|\right||f|\right\|_{A}\right.\right.\right.\right.\right.$. Thus $\Gamma_{m, A}(K) \leq C R_{m, A}^{\prime}(G)$, for all $G \supset K$.

From [10], $R_{m, A}^{\prime}$ is an outer capacity, and the result follows.

For $f \geq 0, \mathcal{R}_{m} * f$ is lower semi-continuous. Hence the set $X_{t}=$ $\left\{x: \mathcal{R}_{m} * f \geq t\right\}$ is a $G_{\delta^{-}}$set and by [6] it is capacitable since $A$ and $A^{*}$ verify the $\Delta_{2}$ condition. Consequently, there is a positive constant, independent of $X_{t}$, such that

$$
C^{-1} R_{m, A}^{\prime}\left(X_{t}\right) \leq \Gamma_{m, A}\left(X_{t}\right) \leq C R_{m, A}^{\prime}\left(X_{t}\right)
$$


Remark 3.2. Let $f \in D\left(R^{N}\right)$. Since $Y_{t}=\left\{x: \mathcal{G}_{m} * f \geq t\right\}$ is a compact set, we know from [8] that there is a positive constant $C$, independent of $Y_{t}$, such that

$$
C^{-1} R_{m, A}^{\prime}\left(Y_{t}\right) \leq B_{m, A}^{\prime}\left(Y_{t}\right) \leq C R_{m, A}^{\prime}\left(Y_{t}\right) .
$$

Hence, there is a positive constant $C$, independent of $Y_{t}$, such that

$$
C_{m, A}^{-1} \Gamma\left(Y_{t}\right) \leq B_{m, A}^{\prime}\left(Y_{t}\right) \leq C \Gamma_{m, A}\left(Y_{t}\right)
$$

Let $\mathcal{S}_{m}$ as above and set

$$
\mathcal{S}_{m, A}^{\prime}(X)=\inf \left\{\||| f \mid\|_{A}: f \in \mathbf{L}_{A}^{+} \text {and } \mathcal{S}_{m} * f \geq 1 \text { on } X\right\} .
$$

Theorem 3.7. Let $A$ be an $N$-function such that $A$ and $A^{*}$ verify the $\Delta_{2}$ condition and $m$ a positive integer. Then there is a constant $C$ depending only on $N, m$ and $A$ such that for all $f \in \mathbf{L}_{A}^{+}$

$$
\int_{0}^{\infty} \mathcal{S}_{m, A}^{\prime}\left(\left\{x: \mathcal{S}_{m} * f(x) \geq t\right\}\right) d t \leq C\left|\|f \mid\|_{A} .\right.
$$

Proof. Since $A$ verifies the $\Delta_{2}$ condition, there is a sequence $\left(f_{i}\right)_{i} \subset$ $D\left(R^{N}\right)$ such that \|\|$f-f_{i}\|\|_{A} \rightarrow 0$ as $i \rightarrow \infty$. Hence

$$
\left\{x: \mathcal{S}_{m} * f(x) \geq 2 t\right\} \subset\left\{x: \mathcal{S}_{m} *\left|f-f_{i}\right|(x) \geq t\right\} \cup\left\{x: \mathcal{S}_{m} * f_{i}(x) \geq t\right\} .
$$

Thus

$$
\mathcal{S}_{m, A}^{\prime}\left(\left\{x: \mathcal{S}_{m} * f(x) \geq 2 t\right\}\right) \leq\|\| \frac{f-f_{i}}{t} \|_{A}+\mathcal{S}_{m, A}^{\prime}\left(\left\{x: \mathcal{S}_{m} * f_{i}(x) \geq t\right\}\right) .
$$

So

$$
\lim _{i \rightarrow \infty} \mathcal{S}_{m, A}^{\prime}\left(\left\{x: \mathcal{S}_{m} * f_{i}(x) \geq t\right\}\right) \geq \mathcal{S}_{m, A}^{\prime}\left(\left\{x: \mathcal{S}_{m} * f(x) \geq 2 t\right\}\right) .
$$

This implies that it suffices to prove the theorem for $f \in D\left(R^{N}\right)$.

But from Lemma 3.6 and Remark 3.2, it suffices to consider only $\Gamma_{m, A}$ in place of $\mathcal{S}_{m, A}^{\prime}$.

Let $g=\mathcal{S}_{m} * f$. Then 
NOUREDDINE AISSAOUI CAPACITARY TYPE ESTIMATES IN STRONGLY NONLINEAR . .

$$
\begin{aligned}
\int_{0}^{\infty} \Gamma_{m, A}(\{x: g(x) \geq t\}) d t & =\sum_{k=-\infty}^{+\infty} \int_{2^{k}}^{2^{k+1}} \Gamma_{m, A}(\{x: g(x) \geq t\}) d t \\
& \leq \sum_{k=-\infty}^{+\infty} \Gamma_{m, A}\left(\left\{x: g(x) \geq 2^{k}\right\}\right) 2^{k} .
\end{aligned}
$$

Remark that $\left\{x: g(x)>2^{k-1}\right\}$ is a neighborhood of the compact $\left\{x: g(x) \geq 2^{k}\right\}$.

As in [1] define $T(x) \equiv 0$, for $x \leq 0, T(x) \equiv 1$, for $x \geq 1$, and $T \in C^{\infty}(R)$ otherwise.

Let $T_{k}(x)=2^{k} T\left(2^{2-k} x-1\right)$. Then

$$
T_{k} \circ g \in D\left(R^{N}\right) \text { and } 2^{-k} T_{k} \circ g \equiv 1 \text { on }\left\{x: g(x)>2^{k-1}\right\} .
$$

Hence

$$
\begin{gathered}
\sum_{k=-\infty}^{+\infty} \Gamma_{m, A}\left(\left\{x: g(x) \geq 2^{k}\right\}\right) 2^{k} \leq \sum_{k=-\infty|\beta|=m}^{+\infty} \sum_{C\left|\left\|f|\||_{A}\right.\right.}\left\|D^{\beta}\left(T_{k} \circ g\right)\right\| \|_{A} \leq \\
C
\end{gathered}
$$

by Theorem 3.5, since the sequence $\left(T_{k}\right)_{k \in \mathbf{Z}}$ verifies (1).

\section{A space of quasicontinuous functions}

This part is devoted to generalize some results in [2] and in [17] relative to the $\mathbf{L}^{p}$ Lebesgue classes.

From Theorem 3.7, it is natural to seek when the quantity

$$
\int_{0}^{\infty} B_{m, A}^{\prime}(\{x:|\psi| \geq t\}) d t
$$

defines a norm on a linear space of functions $\psi$ on $R^{N}$. Although the answer is not known in general, we shall show that (2) is equivalent to a certain norm of $\psi$.

Definition 4.1. for $\psi$ a function on $R^{N}$, define for $m>0, \mathbf{K}_{\psi}$ and $\Lambda_{m, A}(\psi)$ as

$$
\begin{aligned}
\mathbf{K}_{\psi} & =\left\{f \in \mathbf{L}_{A}^{+}: \mathcal{G}_{m} * f(x) \geq|\psi(x)|, \forall x \in R^{N}\right\} \\
\Lambda_{m, A}(\psi) & =\inf \left\{|||f| \|_{A}: f \in \mathbf{K}_{\psi}\right\} .
\end{aligned}
$$


Proposition 4.1. $\Lambda_{m, A}$ has the following properties

$$
\begin{aligned}
\Lambda_{m, A}\left(\psi_{1}\right) & \leq \Lambda_{m, A}\left(\psi_{2}\right), \text { if }\left|\psi_{1}\right| \leq\left|\psi_{2}\right| ; \\
\Lambda_{m, A}\left(\chi_{E}\right) & =B_{m, A}^{\prime}(E), \text { for all } E ; \\
\Lambda_{m, A}\left(\psi \cdot \chi_{\left(\cup_{j} K_{j}\right)}\right) & \leq \sum_{j} \Lambda_{m, A}\left(\psi \cdot \chi_{K_{j}}\right) .
\end{aligned}
$$

Proof. The first two properties are immediate. For the last one we proceed as in proof of subadditivity of $B_{m, A}^{\prime}$. See [10].

If $\sum_{j} \Lambda_{m, A}\left(\psi \cdot \chi_{K_{j}}\right)=+\infty$, there is nothing to prove.

Let $\sum_{j} \Lambda_{m, A}\left(\psi \cdot \chi_{K_{j}}\right)<\infty$. Then $\Lambda_{m, A}\left(\psi \cdot \chi_{K_{j}}\right)<\infty$, for all $j$. Hence

$\forall \varepsilon>0, \exists f_{j} \in \mathbf{L}_{A}^{+}: \mathcal{G}_{m} * f_{j} \geq\left|\psi \cdot \chi_{K_{j}}(x)\right|$ on $R^{N}$ and $\left\|\left|f_{j}\right|\right\|_{A} \leq$ $\Lambda_{m, A}\left(\psi \cdot \chi_{K_{j}}\right)+\frac{\varepsilon}{2^{j}}$.

We pose $f=\sup _{i} f_{i}$. Then from [10], \|\|$f\left|\left\|_{A} \leq \sum_{j}\left|\left\|f_{j} \mid\right\|_{A}\right.\right.\right.$.

Hence $\||f|\|_{A} \leq \sum_{j} \Lambda_{m, A}\left(\psi \cdot \chi_{K_{j}}\right)+\varepsilon$.

This implies $f \in \mathbf{L}_{A}^{+}$. But $\mathcal{G}_{m} * f \geq\left|\psi \cdot \chi_{\left(\cup_{j} K_{j}\right)}\right|$ on $R^{N}$. Thus

$$
\Lambda_{m, A}\left(\psi \cdot \chi_{\left(\cup_{j} K_{j}\right)}\right) \leq \sum_{j} \Lambda_{m, A}\left(\psi \cdot \chi_{K_{j}}\right) .
$$

The proof is finished.

Proposition 4.2. 1. Let $A$ be any $N$-function and $m \in R^{+}$. The function $\Lambda_{m, A}$ defines a norm on $C_{0}=C_{0}\left(R^{N}\right)$, and

$$
\frac{1}{4} \Lambda_{m, A}(\psi) \leq \int_{0}^{\infty} B_{m, A}^{\prime}(\{x:|\psi(x)| \geq t\}) d t
$$

for all continuous functions $\psi$.

2. Let $A$ be an $N$-function such that $A$ and $A^{*}$ verify the $\Delta_{2}$ condition and $m$ a positive integer. Then there is a constant $C$ such that

$$
\frac{1}{4} \Lambda_{m, A}(\psi) \leq \int_{0}^{\infty} B_{m, A}^{\prime}(\{x:|\psi(x)| \geq t\}) d t \leq C \Lambda_{m, A}(\psi)
$$

for all continuous functions $\psi$.

Proof. 1. If $\psi_{i} \in C_{0}$ and $f_{i} \in \mathbf{K}_{\psi_{i}}$, for $i=1,2$, then $f_{1}+f_{2} \in \mathbf{K}_{\psi_{1}+\psi_{2}}$, and thus 
NOUREDDINE AISSAOUI CAPACITARY TYPE ESTIMATES IN STRONGLY NONLINEAR ...

$$
\Lambda_{m, A}\left(\psi_{1}+\psi_{2}\right) \leq\left|\left\|f_{1}+f_{2}\left|\left\|\left.\right|_{A} \leq\right\|\right|\left|f_{1}\right|\right\|\right|_{A}+\mid\left\|f_{2}\right\| \|_{A} .
$$

Hence

$$
\Lambda_{m, A}\left(\psi_{1}+\psi_{2}\right) \leq \Lambda_{m, A}\left(\psi_{1}\right)+\Lambda_{m, A}\left(\psi_{2}\right)
$$

It is obvious that $\Lambda_{m, A}(0)=0$, and $\Lambda_{m, A}(d \psi)=|d| \Lambda_{m, A}(\psi)$.

Let $\psi \in C_{0}$ be such that $\Lambda_{m, A}(\psi)=0$. Then

$$
\forall \varepsilon>0, \exists f \in \mathbf{L}_{A}^{+}: \mathcal{G}_{m} * f \geq|\psi(x)|, \forall x \in R^{N} \text { and }|||f| \|_{A} \leq \varepsilon .
$$

Recall that $\left\|\left|\mathcal{G}_{m} * f\right|\right\|_{A} \leq\left\|\mathcal{G}_{m}\right\|_{1} \cdot\left|\|f \mid\|_{A}\right.$. See [14 or 22].

Since $C_{0} \subset \mathbf{L}_{A}$, we get \|\|$\psi\|\|_{A} \leq\left\|\left|\mathcal{G}_{m} * f\right|\right\|_{A} \leq\left\|\mathcal{G}_{m}\right\|_{1} \cdot \mid\|f\| \|_{A}=$ \|\|$f \|_{A} \leq \varepsilon$, and $\psi \equiv 0$.

This shows that $\Lambda_{m, A}$ is a norm on $C_{0}$.

Let $j \in Z$ and $\gamma>1$. Set $K_{j}=\left\{x: \gamma^{j} \leq|\psi(x)| \leq \gamma^{j+1}\right\}$, and $E_{j}=$ $\left\{x: \gamma^{j} \leq|\psi(x)|\right\}$.

From Proposition 4.1, it follows that

$$
\Lambda_{m, A}(\psi) \leq \sum_{j \in \mathbf{Z}} \Lambda_{m, A}\left(\psi \cdot \chi_{K_{j}}\right) \leq \sum_{j \in \mathbf{Z}} \gamma^{j+1} B_{m, A}^{\prime}\left(E_{j}\right) .
$$

On the other hand

$$
\int_{0}^{\infty} B_{m, A}^{\prime}(\{x:|\psi(x)| \geq t\}) d t \geq \sum_{j \in \mathbf{Z}} \gamma^{j}\left(1-\gamma^{-1}\right) B_{m, A}^{\prime}\left(E_{j}\right) .
$$

Hence

$$
\Lambda_{m, A}(\psi) \leq \frac{\gamma^{2}}{\gamma-1} \int_{0}^{\infty} B_{m, A}^{\prime}(\{x:|\psi(x)| \geq t\}) d t
$$

The function $h(\gamma)=\frac{\gamma^{2}}{\gamma-1}$ attains the minimum at $\gamma=2$, and $h(2)=4$.

2. Let $f \in \mathbf{K}_{\psi}$. Then by Theorem 3.7, there is a constant $C$ such that

$$
\int_{0}^{\infty} B_{m, A}^{\prime}(\{x:|\psi(x)| \geq t\}) d t \leq\left. C|\| f|\right|_{A}
$$


This completes the proof.

We define a new Banach space, $\mathbf{L}_{A}\left(B_{m, A}^{\prime}\right)$, as the completion of $D\left(R^{N}\right)$ in the norm $\Lambda_{m, A}$.

Lemma 4.3. Let $A$ be an $N$-function such that $A$ and $A^{*}$ verify the $\Delta_{2}$ condition and $m$ a positive integer. Then $\mathbf{L}_{m, A} \subset \mathbf{L}_{A}\left(B_{m, A}^{\prime}\right)$.

Proof. Let $f \in \mathbf{L}_{A}$ and choose a sequence $\left(f_{i}\right)_{i} \subset D\left(R^{N}\right)$ such that $\left\||| f_{i}-f \mid\right\|_{A} \rightarrow 0$ as $i \rightarrow \infty$. Then by Proposition 4.2 and Theorem 3.7

$$
\begin{gathered}
\Lambda_{m, A}\left(\mathcal{G}_{m} * f_{i}-\mathcal{G}_{m} * f\right) \leq \\
4 \int_{0}^{\infty} B_{m, A}^{\prime}\left(\left\{x: \mathcal{G}_{m} *\left|f_{i}(x)-f(x)\right| \geq t\right\}\right) d t \leq C \mid\left\|f_{i}-f\right\| \|_{A} .
\end{gathered}
$$

Hence $\mathcal{G}_{m} * f_{i}-\mathcal{G}_{m} * f \rightarrow 0$ in $\mathbf{L}_{A}\left(B_{m, A}^{\prime}\right)$.

Define $\mathcal{G}_{m, R}(x)=\mathcal{G}_{m}(x)$ for $|x| \leq R$ and $\mathcal{G}_{m, R}(x)=0$ for $|x|>R$.

Then $\mathcal{G}_{m, R} * f_{i} \in D\left(R^{N}\right)$ and

$$
\left(\mathcal{G}_{m}-\mathcal{G}_{m, R}\right) *\left|f_{i}\right|(x) \leq\left\|f_{i}\right\|_{\infty} \int_{|y|>R} \mathcal{G}_{m}(y) d y \searrow 0, \text { as } R \rightarrow \infty .
$$

This implies that $\mathcal{G}_{m} * f_{i}$, and thus $\mathcal{G}_{m} * f$ belongs to $\mathbf{L}_{A}\left(B_{m, A}^{\prime}\right)$.

Lemma 4.4. Let $A$ be an $N$-function such that $A$ and $A^{*}$ verify the $\Delta_{2}$ condition and $m$ a positive integer. Any continuous compactly supported function belongs on $\mathbf{L}_{A}\left(B_{m, A}^{\prime}\right)$.

Proof. Let $f$ be such function. Then there is a sequence $\left(f_{i}\right)_{i} \subset D\left(R^{N}\right)$ supported in a fixed ball such that $\left(f_{i}\right)_{i}$ converges uniformly to $f$. Hence by Lebegue's Theorem, $\int A\left(\left|f-f_{i}\right|(x)\right) d x \rightarrow 0$. Since $A$ verifies the $\Delta_{2}$ condition, $\left|\left\|f_{i}-f|\||_{A} \rightarrow 0\right.\right.$. But there is $\varphi \in \mathbf{L}_{A}^{+}$such that $f_{i}-f=\mathcal{G}_{m} * \varphi$. Hence

$$
\Lambda_{m, A}\left(f_{i}-f\right) \leq\|\| \psi\|\|_{A}=\left|\left\|f_{i}-f|\||_{A} .\right.\right.
$$

The proof is complete.

Lemma 4.5. Let $A$ be an $N$-function such that $A$ and $A^{*}$ verify the $\Delta_{2}$ condition and $m$ a positive integer. If $\psi \in C$ is such that

$$
\int_{0}^{\infty} B_{m, A}^{\prime}(\{x:|\psi(x)| \geq t\}) d t<\infty,
$$

then $\psi \in \mathbf{L}_{A}\left(B_{m, A}^{\prime}\right)$. 
Proof. Let $\psi \in C$ be such that $\int_{0}^{\infty} B_{m, A}^{\prime}(\{x:|\psi(x)| \geq t\}) d t<\infty$.

By Proposition 4.2 we have $\Lambda_{m, A}(\psi)<\infty$. So there is an $f \in \mathbf{L}_{A}^{+}$ such that $\mathcal{G}_{m} * f \geq|\psi(x)|$ on $R^{N}$.

Let $h \in C_{0}$ be arbitrary, and let $\eta \in C_{0}$ be a cut off function such that $\eta(x)=1$ on supph, and $0 \leq \eta \leq 1$. Thus $\eta \psi \in C_{0}$, and $(1-\eta) h=0$.

Hence $|\psi(x)-\eta(x) \psi(x)| \leq(1-\eta(x))\left|\mathcal{G}_{m} * f(x)-h(x)\right|$.

Then from Proposition 4.1, $\Lambda_{m, A}(\psi-\eta \psi) \leq \Lambda_{m, A}\left(\mathcal{G}_{m} * f-h\right)$.

From Lemma 4.4, $\eta \psi \in \mathbf{L}_{A}\left(B_{m, A}^{\prime}\right)$.

So, for $\varepsilon>0$, there is $v \in D\left(R^{N}\right)$ such that $\Lambda_{m, A}(v-\eta \psi) \leq \frac{\varepsilon}{2}$.

And from Lemma $4.3, \mathcal{G}_{m} * f \in \mathbf{L}_{A}\left(B_{m, A}^{\prime}\right)$.

So, there is $h \in D\left(R^{N}\right)$ such that $\Lambda_{m, A}\left(\mathcal{G}_{m} * f-h\right) \leq \frac{\varepsilon}{2}$.

Now $\Lambda_{m, A}(\psi-v) \leq \Lambda_{m, A}(\psi-v \psi)+\Lambda_{m, A}(v-\eta \psi) \leq \varepsilon$.

Theorem 4.6. Let $A$ be an $N$-function such that $A$ and $A^{*}$ verify the $\Delta_{2}$ condition and $m$ a positive integer. Then a function $\psi$ on $R^{N}$ belongs to $\mathbf{L}_{A}\left(B_{m, A}^{\prime}\right)$ if and only if it is $(m, A)$-quasicontinuous, and

$$
\int_{0}^{\infty} B_{m, A}^{\prime}(\{x:|\psi(x)| \geq t\}) d t<\infty
$$

Proof. Let $\psi \in \mathbf{L}_{A}\left(B_{m, A}^{\prime}\right)$. Then there is a sequence $\left(\psi_{i}\right)_{i}$ of continuous, compactly supported functions such that

$$
\int_{0}^{\infty} B_{m, A}^{\prime}\left(\left\{x:\left|\psi(x)-\psi_{i}(x)\right| \geq t\right\}\right) d t \leq 4^{-i} .
$$

This implies

$$
B_{m, A}^{\prime}\left(\left\{x:\left|\psi(x)-\psi_{i}(x)\right| \geq 2^{-i}\right\}\right) \cdot 2^{-i} \leq 4^{-i} .
$$

Hence

$$
B_{m, A}^{\prime}\left(\left\{x:\left|\psi(x)-\psi_{i}(x)\right| \geq 2^{-i}\right\}\right) \leq 2^{-i} .
$$
that

Since $B_{m, A}^{\prime}$ is an outer capacity (see[10]), there is an open set such

$$
O_{i} \supset\left\{x:\left|\psi(x)-\psi_{i}(x)\right| \geq 2^{-i}\right\} \text { and } B_{m, A}^{\prime}\left(O_{i}\right) \leq 2^{-i+1} .
$$


NOUREDDINE AISSAOUI CAPACITARY TYPE ESTIMATES IN STRONGLY NONLINEAR . .

$$
\text { Pose } O=\bigcup_{i=k}^{\infty} O_{i} \text {. Then } B_{m, A}^{\prime}(O) \leq \sum_{i=k}^{\infty} B_{m, A}^{\prime}\left(O_{i}\right) \leq 2^{-k+2} \text {. }
$$

Moreover $\psi_{i}(x) \rightarrow \psi(x)$ uniformly on $O^{c}$, and $k$ is arbitrary. Thus $\psi$ is $(m, A)$-quasicontinuous on $R^{N}$.

Let $\psi$ be an $(m, A)$-quasicontinuous function that satisfies (4). We can assume that $\psi$ is real valued. Define, for $R>0, \psi_{R}$ by $\psi_{R}(x)=\psi(x)$ when $|\psi(x)| \leq R, \psi_{R}(x)=R$ when $\psi(x)>R$, and $\psi_{R}(x)=-R$ when $\psi(x)<-R$. Then $\psi_{R}$ is also quasicontinuous, and

$$
\begin{gathered}
\int_{0}^{\infty} B_{m, A}^{\prime}\left(\left\{x:\left|\psi(x)-\psi_{R}(x)\right| \geq t\right\}\right) d t= \\
\int_{0}^{\infty} B_{m, A}^{\prime}(\{x:|\psi(x)| \geq R+t\}) d t \\
\leq \int_{R}^{\infty} B_{m, A}^{\prime}(\{x:|\psi(x)| \geq t\}) d t
\end{gathered}
$$

which is arbitrarily small.

Since $\psi_{R}$ is quasicontinuous, for $\varepsilon>0$, we can find an open set $O$ with $B_{m, A}^{\prime}(O)<\varepsilon$ such that $\psi_{R}$ restricted to $O^{c}$ is continuous. By the Tietze extension theorem there is a continuous function $\varphi$ such that $\left.\varphi\right|_{O^{c}}=\left.\psi_{R}\right|_{O^{c}}$ and $\|\varphi\|_{\infty} \leq R$. Then

$$
\int_{0}^{\infty} B_{m, A}^{\prime}\left(\left\{x:\left|\varphi(x)-\psi_{R}(x)\right| \geq t\right\}\right) d t \leq 2 R B_{m, A}^{\prime}(O)<2 R \varepsilon
$$

and the proof is finished.

Corollary 4.7 Let $A$ and $m$ be as in the previous theorem. If $\psi \in$ $\mathbf{L}_{A}\left(B_{m, A}^{\prime}\right)$, and if $\varphi$ is an $(m, A)$-quasicontinuous function such that $|\varphi| \leq|\psi|$ a.e., then

$$
\varphi \in \mathbf{L}_{A}\left(B_{m, A}^{\prime}\right) \text {, and }\|\varphi\|_{\mathbf{L}_{A}\left(B_{m, A}^{\prime}\right)} \leq\|\psi\|_{\mathbf{L}_{A}\left(B_{m, A}^{\prime}\right)} .
$$

Moreover, if $\mathbf{L}_{m, A}$ is imbedded in a Banach space $\mathbf{B}$ such that $\|\cdot\|_{\mathbf{B}}$ is monotone in the sense that $\|u\|_{\mathbf{B}} \leq\|v\|_{\mathbf{B}}$ for all $u$ and $v$ such that $|u(x)| \leq|v(x)|$ everywhere, then $\mathbf{B}$ contains $\mathbf{L}_{A}\left(B_{m, A}^{\prime}\right)$.

Proof. By [9] the assumptions imply that $|\varphi| \leq|\psi|(m, A)$-q.e. It follows that $\varphi$ verifies (4), and thus $\varphi \in \mathbf{L}_{A}\left(B_{m, A}^{\prime}\right)$. An easy extension of (3) gives the norm inequality.

For the second part, suppose that $\|u\|_{\mathbf{B}} \leq C\|u\|_{m, A}$ for all $v \in \mathbf{L}_{m, A}$. Let $v \in D\left(R^{N}\right)$. Then $\|v\|_{\mathbf{B}} \leq\left\|\mathcal{G}_{m} * f\right\|_{\mathbf{B}} \leq C\left\|\mathcal{G}_{m} * f\right\|_{\mathbf{B}}=C \mid\|f\| \|_{A}$ for all $f \in \mathbf{K}_{v}$. Hence $\|v\|_{\mathbf{B}} \leq C \Lambda_{m, A}(v)$.

Since $\mathbf{L}_{A}\left(B_{m, A}^{\prime}\right)$ is the closure of $D\left(R^{N}\right)$, the conclusion follows.

We describe now the dual space to $\mathbf{L}_{A}\left(B_{m, A}^{\prime}\right)$. 
Theorem 4.8. Let $A$ be an $N$-function such that $A$ verifies the $\Delta_{2}$ condition and $m>0$. Then the dual space $\mathbf{L}_{A}\left(B_{m, A}^{\prime}\right)^{*}$ can be identified with the space of all $\mu \in \mathfrak{M}\left(R^{N}\right)$ such that $\mathcal{G}_{m} *|\mu| \in \mathbf{L}_{A^{*}}$. If $\psi \in$ $\mathbf{L}_{A}\left(B_{m, A}^{\prime}\right)$ and $\mu \in \mathbf{L}_{A}\left(B_{m, A}^{\prime}\right)^{*}$, then $\psi \in \mathbf{L}^{1}(|\mu|)$, and the duality is given by

$$
\langle\mu, \psi\rangle=\int_{R^{N}} \psi d \mu .
$$

Moreover, the norm of $\mu$ in $\mathbf{L}_{A}\left(B_{m, A}^{\prime}\right)^{*}$ is $\left\|\mathcal{G}_{m} *|\mu|\right\|_{A^{*}}$.

Proof. Let $\mu \in \mathfrak{M}\left(R^{N}\right)$ be such that $\left\|\mathcal{G}_{m} *|\mu|\right\|_{A^{*}}<\infty$, and $\psi \in C_{0}$. Let $f \in \mathbf{K}_{\psi}$. Then

$$
\begin{gathered}
\int_{R^{N}}|\psi| d|\mu| \leq \int_{R^{N}}\left(\mathcal{G}_{m} * f\right) d|\mu|=\int_{R^{N}} f\left(\mathcal{G}_{m} *|\mu|\right) d x \leq \\
\left\|f\left|\left\|_{A} \cdot\right\| \mathcal{G}_{m} *\right| \mu \mid\right\|_{A^{*}}
\end{gathered}
$$

Hence

$$
\int_{R^{N}}|\psi| d|\mu| \leq \Lambda_{m, A}(\psi) \cdot\left\|\mathcal{G}_{m} *|\mu|\right\|_{A^{*}}
$$

This implies that any Cauchy sequence in $\mathbf{L}_{A}\left(B_{m, A}^{\prime}\right)$ is Cauchy in $\mathbf{L}^{1}(|\mu|)$ and thus $\mathbf{L}_{A}\left(B_{m, A}^{\prime}\right) \subset \mathbf{L}^{1}(|\mu|)$ since $\mathbf{L}_{A}\left(B_{m, A}^{\prime}\right)$ is the completion of $C_{0}$. Moreover

$$
\int_{R^{N}} \psi d \mu \leq|||f|\left\|\left.\right|_{A} \cdot\right\| \mathcal{G}_{m} *|\mu| \|_{A^{*}}
$$

so $\mu$ defines a linear functional on $\mathbf{L}_{A}\left(B_{m, A}^{\prime}\right)$ with norm $\|\mu\| \leq$ $\left\|\mathcal{G}_{m} *|\mu|\right\|_{A^{*}}$.

Conversely, consider $\mu: \psi \mapsto\langle\mu, \psi\rangle$ a bounded linear functional on $\mathbf{L}_{A}\left(B_{m, A}^{\prime}\right)$ with norm $\|\mu\|$. Let $K$ be a compact set, and let $\chi \in C_{0}$ such that $0 \leq \chi \leq 1$, and $\left.\chi\right|_{K}=1$. Then for any $\psi \in C_{0}(K)$ we have $|\psi| \leq\|\psi\|_{\infty} \cdot \chi$, and thus

$$
|\langle\mu, \psi\rangle| \leq\|\mu\| \cdot \Lambda_{m, A}(\psi) \leq\|\mu\| \cdot\|\psi\|_{\infty} \cdot \Lambda_{m, A}(\chi) .
$$

This implies that $\mu$ is a bounded linear functional on $C_{0}(K)$ for each compact $K$. By the Riesz representation theorem, $\mu$ can be identified with a Radon measure so that $\langle\mu, \psi\rangle=\int_{R^{N}} \psi d \mu$ for all $\psi \in C_{0}$. It only remains to prove that $\left\|\mathcal{G}_{m} *|\mu|\right\|_{A^{*}} \leq\|\mu\|$ since $C_{0}$ is dense in $\mathbf{L}_{A}\left(B_{m, A}^{\prime}\right)$.

We have 


$$
\int_{R^{N}} \psi d|\mu|=\sup \left\{\int_{R^{N}} \varphi d \mu: \varphi \in C_{0},|\varphi| \leq \psi\right\} .
$$

By assumption $\left|\int_{R^{N}} \varphi d \mu\right| \leq\|\mu\| . \Lambda_{m, A}(\varphi)$, and thus $\int_{R^{N}} \psi d|\mu| \leq$ $\|\mu\| . \Lambda_{m, A}(\psi)$ for all positive $\psi$ in $C_{0}$.

By approximation from below, here $C_{0}$ is dense in $\mathbf{L}_{A}$ because $A$ verifies the $\Delta_{2}$ condition, it follows that for all $f \in \mathbf{L}_{A}^{+}$

$$
\int_{R^{N}} f\left(\mathcal{G}_{m} *|\mu|\right) d x=\int_{R^{N}} \underset{\|\mu\| .\|f \mid\| f \|_{A} .}{\left.f\left(\mathcal{G}_{m} *|\mu|\right) d x \leq\|\mu\| . \Lambda_{m, A}\left(\mathcal{G}_{m} * f\right)\right) \leq}
$$

But we know that

$$
\left\|\mathcal{G}_{m} *|\mu|\right\|_{A^{*}}=\sup \left\{\int_{R^{N}} f\left(\mathcal{G}_{m} *|\mu|\right) d x:\left|\|f \mid\|_{A} \leq 1\right\},\right.
$$

so

$$
\left\|\mathcal{G}_{m} *|\mu|\right\|_{A^{*}} \leq\|\mu\|
$$

This completes the proof.

\section{Maximal operators and capacity}

Let $\left(\theta_{j}\right)_{j}$ be a sequence of convolution operators. Define the maximal operator $J$ by $J(f)=\sup _{j}\left|\theta_{j} * f\right|$, where $f$ is initially taken to be in the Schwarz class of rapidly decreasing $C^{\infty}$ functions on $R^{N}$ denoted by $S=S\left(R^{N}\right)$.

An operator $H: \mathbf{L}_{A} \rightarrow \mathbf{L}_{A}$ is of strong type $(A, A)$ if

$$
\left\|\left|H(f)\|\|_{A} \leq C\right|\right\| f|\||_{A}, \forall f \in \mathbf{L}_{A},
$$

where $C$ is a constant dependent only on $A$.

For more details, see [25].

Definition 5.1. An operator $H: \mathbf{L}_{A} \rightarrow \mathbf{L}_{A}$ is of capacitary weak type $(A, A)$ if

$$
\forall f \in \mathbf{L}_{A}, \forall t>0, R_{m, A}^{\prime}\left(\left\{x: H\left(\mathcal{R}_{m} * f\right)(x) \geq t\right\}\right) \leq C_{A} \frac{\|f \mid\|_{A}}{t},
$$

where $C_{A}$ is a constant dependent only on $N, m$ and $A$. 
NOUREDDINE AISSAOUI CAPACITARY TYPE ESTIMATES IN STRONGLY NONLINEAR . .

$H$ is of capacitary strong type $(m, A)$ if

$$
\forall f \in \mathbf{L}_{A}, \quad \int_{0}^{\infty} R_{m, A}^{\prime}\left(\left\{x: H\left(\mathcal{R}_{m} * f\right)(x) \geq t\right\}\right) d t \leq C\left|\left\|f|\||_{A}\right.\right.
$$

where $C$ is a constant dependent only on $N, m$ and $A$.

Proposition 5.1. 1. Let $A$ be an $N$-function such that $A$ and $A^{*}$ verify the $\Delta_{2}$ condition, $\alpha=\alpha(A)$ and $m$ is a positive integer such that $m<N / \alpha$. If $J$ is of strong type $(A, A)$, then it is also of capacitary strong type $(m, A)$.

2. Let $A$ be any $N$-function. If $J$ is of strong type $(A, A)$ and $0<m<N$, then it is also of capacitary weak type $(m, A)$.

Proof. Let $f \in S$. Then $\theta_{j} *\left(\mathcal{R}_{m} * f\right)=\mathcal{R}_{m} *\left(\theta_{j} * f\right)$.

This implies $J\left(\mathcal{R}_{m} * f\right) \leq \mathcal{R}_{m} *(J(f))$.

By Theorem 3.7,

$$
\int_{0}^{\infty} R_{m, A}^{\prime}\left(\left\{x: J\left(\mathcal{R}_{m} * f\right)(x) \geq t\right\}\right) d t \leq C\left|\| J ( f ) \| \left\|_{A} \leq C\left|\|f \mid\| \|_{A} .\right.\right.\right.
$$

For the second statement, we have the obvious inequality

$$
R_{m, A}^{\prime}\left(\left\{x: \mathcal{R}_{m} * f(x) \geq t\right\}\right) \leq \frac{\||f|\|_{A}}{t} .
$$

Hence

$$
R_{m, A}^{\prime}\left(\left\{x: J\left(\mathcal{R}_{m} * f\right)(x) \geq t\right\}\right) \leq C \frac{\||f|\|_{A}}{t} .
$$

The proof is finished.

\section{Some open questions}

1. The first natural question is: whether or not Theorem 3.7 is valid for $m \in R^{+}$.

2. We recall the definition of radially decreasing convolution kernel. 
Definition 6.1. A function $g$ defined on $R^{N} \times R^{N}$ is a radially decreasing convolution kernel if $g(x, y)=g_{0}(|x-y|)$, where $g_{0}$ is a positive lower semicontinuous, non-increasing function on $R^{+}$and such that $\int_{0}^{1} g_{0}(t) t^{N-1} d t<\infty$.

As in Lebesgue case, can Theorem 3.7 be extended to radially decreasing convolution kernels?

3. For $\mathbf{L}^{p}$ Lebesgue case, we have the obvious capacitary weak inequality

$$
\mathcal{S}_{m, p}\left(\left\{x: \mathcal{S}_{m} * f(x) \geq t\right\}\right) \leq\left(\frac{\|f\|_{p}}{t}\right)^{p}=\frac{\|f\|_{p}^{p}}{t^{p}}
$$

Here $\mathcal{S}_{m, p}(X)=\inf \left\{\|f\|_{p}^{p}: f \in \mathbf{L}_{p}^{+}\right.$and $\mathcal{S}_{m} * f \geq 1$ on $\left.X\right\}$

Hence

$$
\mathcal{S}_{m, p}\left(\left\{x: \mathcal{S}_{m} * f(x) \geq t\right\}\right) \cdot t^{p} \leq\|f\|_{p}^{p} .
$$

And we know the following stronger version of Theorem 3.7

$$
\int_{0}^{\infty} \mathcal{S}_{m, p}\left(\left\{x: \mathcal{S}_{m} * f(x) \geq t\right\}\right) d t^{p} \leq C\|f\|_{p}^{p}
$$

For Orlicz case, we have the obvious capacitary weak inequality

$$
\mathcal{S}_{m, A}\left(\left\{x: \mathcal{S}_{m} * f(x) \geq t\right\}\right) \leq A\left(\frac{\|\| f\|\|_{A}}{t}\right) .
$$

Then the question is: whether or not the following stronger version of Theorem 3.7 holds

$$
\int_{0}^{\infty} \mathcal{S}_{m, A}\left(\left\{x: \mathcal{S}_{m} * f(x) \geq t\right\}\right) d A(t) \leq C A\left(\||f|\|_{A}\right) .
$$

Here $d A(t)=a(t) d t$, and $\mathcal{S}_{m, A}(X)=A\left(\mathcal{S}_{m, A}^{\prime}(X)\right)$.

\section{References}

[1] D. R. Adams, On the existence of capacitary strong type estimates in $R^{N}$. Ark. Mat., 14 (1976), 125-140.

[2] D. R. Adams, Sets and functions of finite $L^{p}$-capacity, Indiana Univ. Math. J., 27 (1978), 611-627. 
NOUREDDINE AISSAOUI CAPACITARY TYPE ESTIMATES IN STRONGLY NONLINEAR . .

[3] D. R. Adams, L.I. Hedberg, Function spaces and potential theory. Springer-Verlag, Berlin, Heidelberg, New York, (1996).

[4] D. R. Adams, J.C. Polking, The equivalence of two definitions of capacity, Proc. Amer. Math. Soc., 37 (1973), 529-534.

[5] R. Adams, Sobolev spaces, Acad. Press, New York, London, Toronto, (1975).

[6] N. Aïssaoui, Note sur la capacitabilité dans les espaces d'Orlicz, Ann. Sci. Math. Québec. 19 (1995), 107-113.

[7] N. Aïssaoui, Instability of capacity in Orlicz spaces, Potential Analysis, Vol. 6, No 4 (1997), 327-346.

[8] N. Aïssaoui, Bessel potentials in Orlicz spaces, Revista Matemática Complutense, Vol. 10, No 1 (1997), 55-79.

[9] N. Aïssaoui, Some developments of strongly nonlinear potential theory, Libertas Mathematica, Vol. XIX (1999), 155-170.

[10] N. Aïssaoui, A. Benkirane, Capacités dans les espaces d'Orlicz, Ann. Sci. Math. Québec. 18(1994), No 1, 1-23.

[11] N. Aïssaoui, A. Benkirane, Potentiel non linéaire dans les espaces d'Orlicz, Ann. Sci. Math. Québec. 18 (1994), No 2, 105-118.

[12] N. Aronszajn, f. Mulla, P. Szeptycki, On spaces of potentials connected with $L^{p}$ classes, Ann. Inst. Fourier (Grenoble) 13 (1963), 211-306.

[13] N. Aronszajn, K.T Smith, Theory of Bessel potentials, Ann. Inst. Fourier (Grenoble) 11 (1961), 383-475.

[14] A. Benkirane, J. P. Gossez, An approximation theorem in higher order Orlicz-Sobolev spaces and applications, Studia Math, 92 (1989), 231-255.

[15] A. P. Calderón, Lebesgue spaces of differentiable functions and distributions, Proc. Sympos.Pure Math., Vol. 4, 33-49, Amer. Math. Soc., Providence, R. I., (1961).

[16] D. Gallardo, Orlicz spaces for which the Hardy-Littlewood maximal operator is bounded, Publicacions Matemàtiques, Vol. 32 (1988), 261-266.

[17] K. Hansson, Imbedding theorems of Sobolev type in potential theory, Math. Scand. 45 (1979), 77-102.

[18] V. P. Havin, V. G. Maz'ya, Non-linear potential theory, Uspehi Math. Nauk., 27:6 (1972), 67-138.

[19] M. A. Krasnosel'skii, Y. B. Rutickii, Convex functions and Orlicz spaces, P. Noordhoff, Groningen, (1961). 
NOUREDDINE AISSAOUI CAPACITARY TYPE ESTIMATES IN STRONGLY NONLINEAR . .

[20] A. Kufner, O. John, S. Fučík, function spaces. Academia, Prague, (1977).

[21] W. A. J. Luxemburg, Banach function spaces. Thesis, Delft University, $(1955)$

[22] R. O'Neil, fractional integration in Orlicz spaces, Trans. Amer. Math. Soc., 115 (1965), 300-328.

[23] M. M. Rao, Z. D. Ren, Theory of Orlicz spaces, Dekker Inc, New York, (1991)

[24] E. M. Stein, Singular integrals and differentiability properties of functions, Princeton Univ. Press, (1970)

[25] A. Torchinsky, Interpolation of operations and Orlicz classes, Studia Math., 59 (1976), 177-207.

Ecole Normale Supérieure

B.P.5206

Ben Souda, Fès

Maroc

E-mail: n.aissaoui@caramail.com

Recibido: 10 de Abril de 2000

Revisado: 30 de Septiembre de 2000 